\title{
EXPERIMENTAL VERIFICATION FOR DIFFERENTIAL EQUATIONS OF SWEEP LINE*
}

\author{
Otohiko Suzuki** \\ (Received July 17, 1962)
}

In the preceding paper," differential equations about the form and tension of sweep line were dealt with.

In order to verify the theory, an experimental study has been carried out and the results obtained are discussed in the present paper. Moreover, the validity of the assumption, appeared in the theory, that the tangential and normal components of frictional force per unit length of rope are independent of the direction of motion is also checked experimentally.

\section{Experimental}

The experimental apparatus is essentially similar to that outlined in the previous paper. ${ }^{2)}$ In order to increase the precision in experiments, the apparatus was slightly modified. Efforts were made to minimize the friction of pulleies, through which a hauling thread is connected to the body to be dragged.

Prior to proceed the experimental study concerning the from and tension of rope, it must be of importance to check the validity of the assumption that the tangential and normal components of frictional force per unit length of rope, which have been denoted previously ${ }^{11}$ by $K_{t}$ and $K_{n}$, respectively, are independent of the inclination angle of it. For the sake of simplicity, rigid rods were used instead of flexible rope. Two smoothly painted wooden rods of diameter $0.77 \mathrm{~cm}$. and length $17.50 \mathrm{~cm}$, and a bamboo rod were combined to form a frame of an isosceles triangle as shown in Fig. 1. If this triangular frame is pulled on a horizontal sand plane, by a thin thread connected to the apex $A$ made by two wooden rods, it moves to the towing direction, canceling the lifts acting on two wooden rods. As the rods were connected each other by means of aluminium pipes, which could be easily

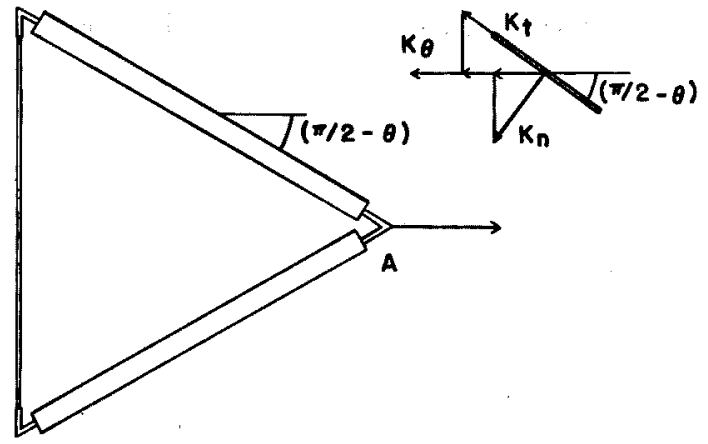

Fig. 1. Showing a triangular frame of wooden rods.

* Studies on the kinematic behaviour of the ground rope of the trawl net-IV.

** Department of Fisheries, Kyoto University, Maizuru (鈴木乙彦, 京大 ·水産) 
bent, the apex angle could be varied arbitrarily according to the length of the bamboo rod. Thus, the drag acting on wooden rods was measured at the towing speed of about $1 \mathrm{~cm} . / \mathrm{sec}$, with attack angles varied at every $10^{\circ}$ between 0 and $90^{\circ}$. Here, the thin bamboo rod had no influence upon this measurement, as the rod did not contact with the sand surface.

Now, we proceed to the next problem. In the whole course of experiments, a uniform flexible nylon twine of $3 \mathrm{~mm}$. in diameter was used as a model of sweep line. The twine was laid on a horizontal plane of dry sand in such a form as shown in Fig. 1 of the reference, ${ }^{1)}$ letting $\alpha=\pi / 2, \overline{\mathrm{AB}}=80 \mathrm{~cm}$. and $\overline{\mathrm{BC}}=100 \mathrm{~cm}$. Then, the end of twine was dragged by a thin thread at slow speeds in the range of 1.23 to $1.66 \mathrm{~cm}$. $/ \mathrm{sec}$. At a regular interval $5 \mathrm{~cm}$. of dragged distance, the towing force was measured and the pattern assumed by the twine was photographed simultaneously from the top. We thus obtained the experimental data as to the tension and the shape of the twine. On the other hand, the value of $K_{t}$ is measured, by pulling the definite length $100 \mathrm{~cm}$. of twine, which is originally laid along the towing direction.

\section{Results and Discussion}

With the consideration of equilibrium as to the wooden rod mentioned above, the drag $K_{\theta}$, at an attack angle of $(\pi / 2-\theta)$, per unit length of the rod is given by

$$
K_{\theta}=K_{n} \cos \theta+K_{t} \sin \theta \text {. }
$$

Dividing the expression by $K_{n}$, we have

$$
K_{\theta} / K_{n}=\cos \theta+\kappa \sin \theta,
$$

which shows that the ratio $K_{\theta} / K_{n}$ is a function of $\theta$, if $\kappa\left(=K_{t} / K_{n}\right)$ is constant. This expression should be verified experimentally. In the case of the rigid rod, the value of $K_{n}$ is easily obtained. On the other hand, the value of $K_{t}$ is considered to estimate rather largely than its intrinsic value, owing to the sand heap appeared at the front

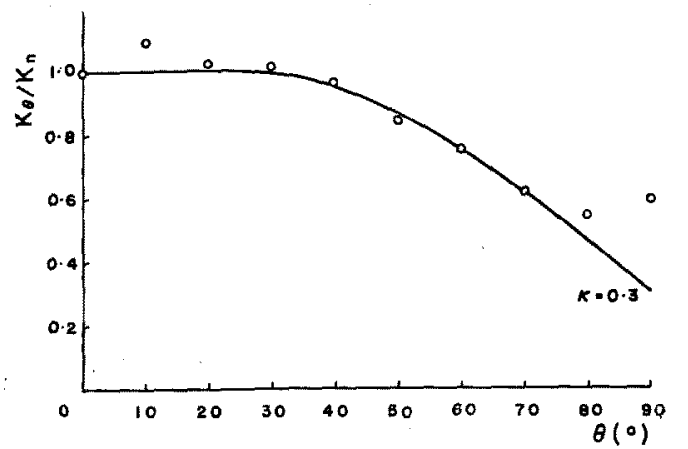

Fig. 2. Relationship between $\theta$ and $K_{\theta} / K_{n}$. Curve shows calculated value for $\kappa=0.3$. end of rod. In Fig. 2 is shown the relation between $\theta$ and the observed value of $K_{\theta} / K_{n}$, an upward trend of plots, as appeared in the range of $\theta$ $=80 \sim 90^{\circ}$, is explained by the above reason. Using the observed value of $K_{\theta} / K_{n}$ for $\theta=60^{\circ}$, where the sand heap is not yet found, we obtain $\kappa=0.3$ in terms of the equation (1). The calculated values of $K_{\theta} / K_{n}$ for this value of $\kappa$ are also illustrated in the same figure. The calculated and the observed value 
agree with each other within experimental error. Consequently, we may consider that the assumption mentioned above is verified in the range of slow speeds.

In the latter experiment, the value of $K_{t}$ for the twine used has been given to be $0.0440 \mathrm{gr} . / \mathrm{cm}$. In order to compare the theoretical with the experimental, it is required to determine the value of $K_{n}$. Contrary to the rigid rod, it is very difficult to measure the value of $K_{n}$ in the case of the flexible twine. Returning to the equations (5) and (6) of the reference, ${ }^{1)}$ and substituting $\theta=\pi / 2$, we have

$$
\left.\frac{Y_{t}}{X_{t}}\right]_{\theta=\pi / 2}=\frac{e^{\pi / 2 . \kappa}-\kappa}{\kappa e^{\pi / 2 \cdot \kappa}+1} .
$$

It is noted that the ratio $\left.Y_{t} / X_{t}\right]_{\theta_{=\pi / 2}}$ should be a constant depending only upon the value of $\kappa$. Accordingly, the plot of $Y_{t}$ against $X_{t}$, experimentally obtained, should give a straight line through the origin, with a slope given by the right-hand member of equation (2). This requirement is found to be satisfied with the data above, as shown in Fig. 3. which gives $\left.Y_{t} / X_{t}\right]_{\theta=\pi / 2}=0.7$. Moreover, from Fig. 4,

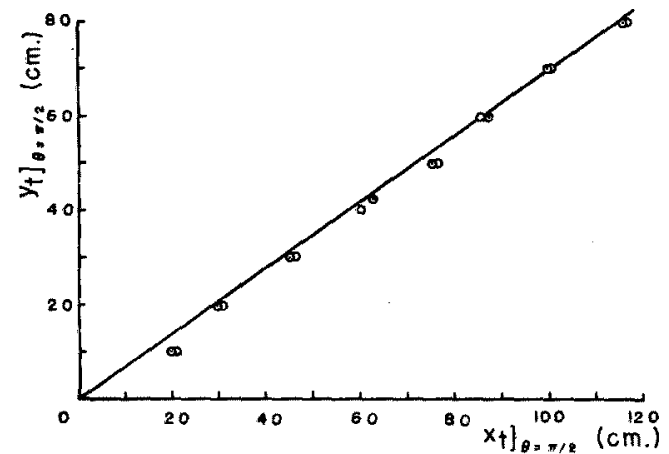

Fig. 3. Plots of $Y_{t}$ against $X_{t}$, experimentally obtained. The slope of straight line gives 0.7 . The marks $\bigcirc$ and $O$ show duplicate experiments.

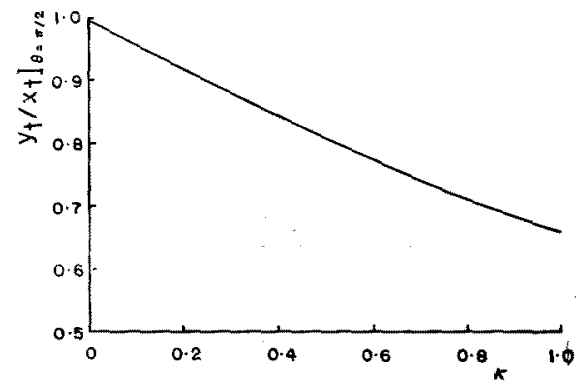

Fig. 4. Ratio $\left.Y_{t} / X_{t}\right]_{\theta=\pi / 2}$ plotted as a function of $\kappa$.

which shows the relation between the value of the right-hand member of equation (2) and $\kappa$, the value of $\kappa$ is found to be 0.85 and thus in turn the value of $K_{n}$ is determined to be $0.0517 \mathrm{gr} / \mathrm{cm}$.

Once the values of $K_{t}$ and $K_{n}$ have been determined, it is possible to compute the values of $T_{0}$ and in turn $T_{t}$ as a function of $l$. Under the experimental condition that $\overline{\mathrm{BC}}=100 \mathrm{~cm}$., the length of the linear part $\overline{\mathrm{RC}}$, which connects the terminal point $R$ of the curve and the rope end $C$ as shown in Fig. 1 of the reference, ${ }^{11}$ and the frictional force acting tangentially upon this part, $K_{t} \times(\overline{\mathrm{RC}})$, are calculated as a function of $l$. Sum of $K_{t} \times(\overline{\mathrm{RC}})$ and $T_{t}$ gives the tension $T_{e}$ at the twine end. If the frictions acting on the thin hauling thread and the pulley are negligibly small as compared with the towing force, the calculated value of $T_{e}$ should agree, in successive stages of towing, with the measured towing force. This requirement is also satisfied as 
shown in Fig. 5, in which the calculated and observed values of towing force $T_{\theta}$, including the calculated values of $T_{0}$ and $T_{t}$, are plotted against $l$. In Fig. 6 . the

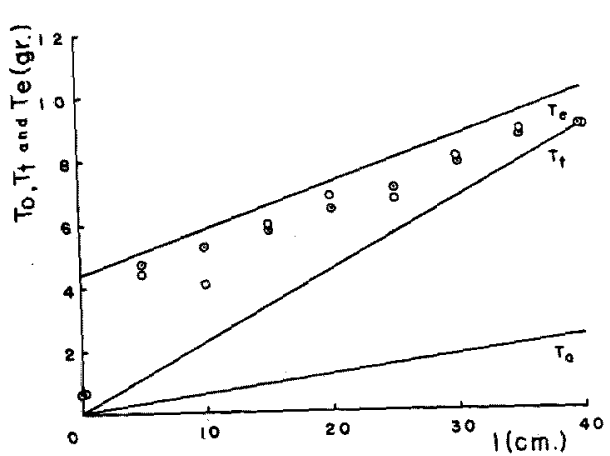

Fig. 5. Calculated and observed values of tension $T_{e}$ plotted against towed distance $l$.

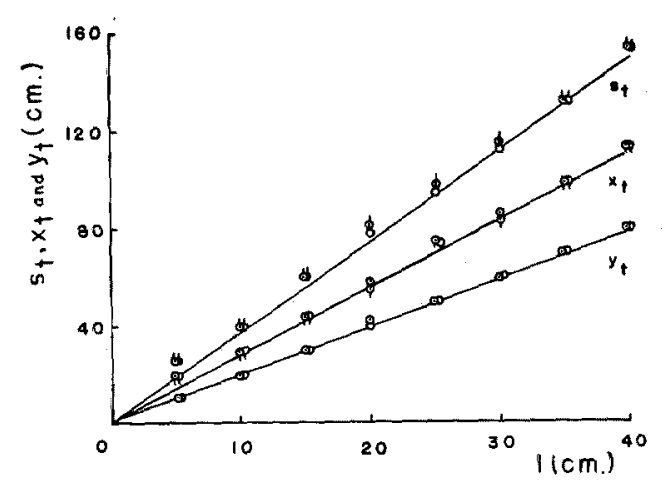

Fig. 6. Characteristic values, $S_{t}, X_{t}$ and $Y_{t}$, of curve plotted as functions of towed distance $l$.

calculated values of $S_{t}, X_{t}$ and $Y_{t}$ are plotted against $l$ together with their experimental ones. They agree with each other, from the nature of fact that the relation between $T_{o} / K_{n}$ and $l$ has been based on the experiment. Morever, as seen in the figure, $S_{t}, X_{t}$ and $Y_{t}$ have linear relations, respectively, against $l$. This means the fact that the patterns assumed by the twine, in the course of towing, are geometrically similar, as pointed out previously."

The author expresses deepest appreciation to Prof. T. Kawakam for his valuable advice and revision.

\section{References}

1) Suzuki, O.: Bull. Jap. Soc. Sei. Fish., 28, 985 (1962).

2) KaWAKaMI, T. and O. SUzUKI: ibid., 25, 413.416 (1959). 